\title{
Multipactor experiment on a dielectric surface
}

\author{
R. B. Anderson, W. D. Getty, ${ }^{\text {a) }}$ M. L. Brake, Y. Y. Lau, ${ }^{\text {b) }}$ R. M. Gilgenbach, \\ and A. Valfells \\ Department of Nuclear Engineering and Radiological Sciences, University of Michigan, Ann Arbor, \\ Michigan 48109-2104
}

(Received 22 January 2001; accepted for publication 1 May 2001)

\begin{abstract}
A novel experiment to investigate single-surface multipactor on a dielectric surface was developed and tested. The compact apparatus consists of a small brass microwave cavity in a high vacuum system. The cavity is $\sim 15 \mathrm{~cm}$ in length with an outer diameter of $\sim 10 \mathrm{~cm}$. A pulsed variable frequency microwave source at $\sim 2.4 \mathrm{GHz}, 2 \mathrm{~kW}$ peak excites the $\mathrm{TE}_{111}$ mode with a strong electric field parallel to a dielectric plate $(\sim 0.2 \mathrm{~cm}$ thickness $)$ that is inserted at midlength of the cavity. The microwave pulses are monitored by calibrated microwave diodes. An electron probe measures electron current and provides temporal measurements of the multipactor electron current with respect to the microwave pulses. Phosphor on the dielectric surface is used to detect multipactor electrons by photoemission. The motivation of this experiment is to test recent theoretical calculations of single-surface multipactor on a dielectric. (c) 2001 American Institute of Physics. [DOI: 10.1063/1.1380687]
\end{abstract}

\section{INTRODUCTION}

Multipactor is a low-pressure electron multiplication phenomenon. It has been known to cause vacuum window failure, generation of excessive noise in communication satellites, detuning of resonant cavities, and increased outgassing of surfaces which leads to more destructive plasma discharges. ${ }^{1-7}$ Detuning of cavities and the breakdown of dielectric windows continue to be undesirable problems in the accelerator community. ${ }^{8-11}$ Two-surface multipactor occurs in a metallic gap under resonant conditions and high power microwaves, ${ }^{4,12}$ while the single-surface multipactor on a dielectric does not require a resonant condition. ${ }^{7,13,14}$ Kinematic calculations have been carried out in the past, along with experimental verifications. ${ }^{15-22}$ However, in recent years, Lau and his collaborators have considered the dynamic evolution of both the two surface multipactor and the multipactor on a dielectric surface..$^{4-7,12-14,23-26}$ Specifically, susceptibility curves for single-surface multipactor for various dielectric materials have been constructed theoretically at the University of Michigan. $7,13,14$

This article introduces a novel experiment designed to test the recent theoretical calculations for dielectric surface breakdown. ${ }^{7}$ Most multipactor experiments consist of a large resonant ring waveguide with a megawatt power supply. ${ }^{11,27-30}$ This experiment uses a small brass resonant cavity $\sim 15 \mathrm{~cm}$ in length and $\sim 10 \mathrm{~cm}$ in diameter with a pulsed $\sim 2.4 \mathrm{GHz}, 2 \mathrm{~kW}$ power supply. The motivation of our experiment is to create a controlled environment for multipactor to occur as close to the theoretical parameters as possible. This will allow testing of theoretical calculations and the exploration of the physics behind the problem.

\footnotetext{
${ }^{a}$ Department of Electrical Engineering and Computer Science, University of Michigan, Ann Arbor, MI 48109-2122.

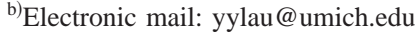

Single-surface multipactor is illustrated in Fig. 1. Initially a stray electron gains energy from the rf electric field $\left(E_{\mathrm{rf}}\right)$ that is parallel to the dielectric surface. If this electron collides with the surface of the dielectric, then one or more secondary electrons are freed from the surface. When the secondary electrons leave the dielectric, a net positive charge is left on the surface of the dielectric. This net positive charge sets up a dc electric field $\left(E_{\mathrm{dc}}\right)$ perpendicular to the dielectric's surface. This $E_{\mathrm{dc}}$ causes the trajectory of the electron to be bent back towards the dielectric. If these electrons gain enough energy from $E_{\mathrm{rf}}$, then they can produce more secondary electrons when they hit the surface and the multiplication process is initiated. Half an rf cycle later, the $E_{\text {rf }}$ reverses direction, leading to severe local heating of the dielectric by electrons. Synchronism between rf field and orbits is not critical in single-surface multipactor because in half an rf cycle there are tens of impacts of multipactor electrons. ${ }^{14}$ This is in sharp contrast to the two-surface multipactor. 4,12

\section{EXPERIMENTAL SETUP}

The microwave cavity (Fig. 2) has a length of $\sim 15 \mathrm{~cm}$ and an outside diameter $\sim 10 \mathrm{~cm}$. This cavity is similar to one designed by Mahoney and Asmussen. ${ }^{31}$ The use of a high- $Q$ resonant cavity provides the large $E_{\text {rf }}$ required for multipactor with the available microwave power. The cavity's dimensions were chosen to preferentially excite the transverse electric $\left(\mathrm{TE}_{111}\right)$ mode. An alumina disk is located near the center of the cavity and held in place by Teflon tabs. The microwave electric field $E_{\mathrm{rf}}$ of the $\mathrm{TE}_{111}$ mode is at a maximum at the center and parallel to the surface of the dielectric disk. Alumina was chosen in this experiment because it has a high secondary emission coefficient and is commonly used as a vacuum window. The disk is positioned where it will not interfere with the antenna and will not 


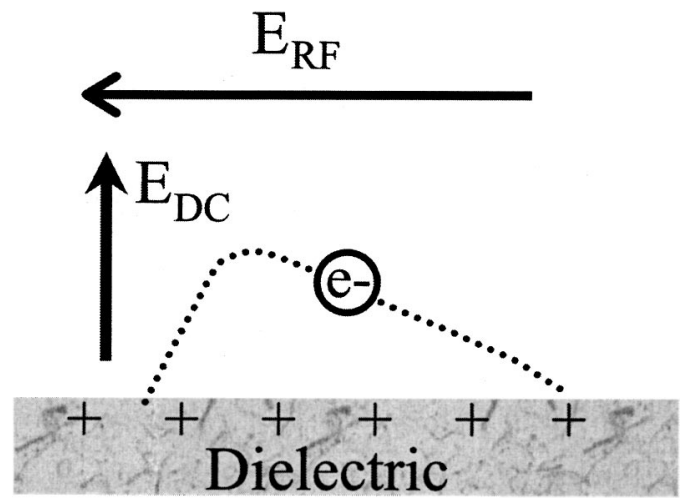

FIG. 1. Multipactor mechanism: If an electron collides with the surface of the dielectric with enough energy, secondary electrons are created. These secondary electrons leave a positive charge on the surface. The same secondaries gain energy from the rf electric field and their trajectory is bent back towards the dielectric due to the dc electric field. If they hit the surface with enough energy, the avalanche continues.

downshift the resonant frequency outside the range of the microwave source. The entire cavity is suspended in a vacuum chamber where the antenna and diagnostics are accessed with vacuum feedthroughs. The vacuum system allows base pressures of $\sim 10^{-6}$ Torr.

A filamentary thermionic emitter was inserted through the array of holes shown on the cylindrical cavity wall in Fig. 2 to provide seed electrons. This was later found to be unnecessary and the emitter was removed.

A pulsed microwave source (EPSCO Microwave Pulse Signal Source, Model: PG5KB) at $\sim 2.4 \mathrm{GHz}$ delivers up to $2 \mathrm{~kW}$ peak power to the resonant cavity. The frequency range of the microwave source is variable between 2.3 and $2.7 \mathrm{GHz}$. The pulse width and repetition rate used were 50 $\mu \mathrm{s}$ and $30 \mathrm{~Hz}$, respectively. The microwave circuit is straightforward and is illustrated in Fig. 3. The power supply is fed into a circulator which is then attached to a dual directional coupler. All microwave components were calibrated with a network analyzer (Hewlett Packard, Model: 8722D). The attenuation depends upon frequency so midband attenuations are used. The dual directional coupler has

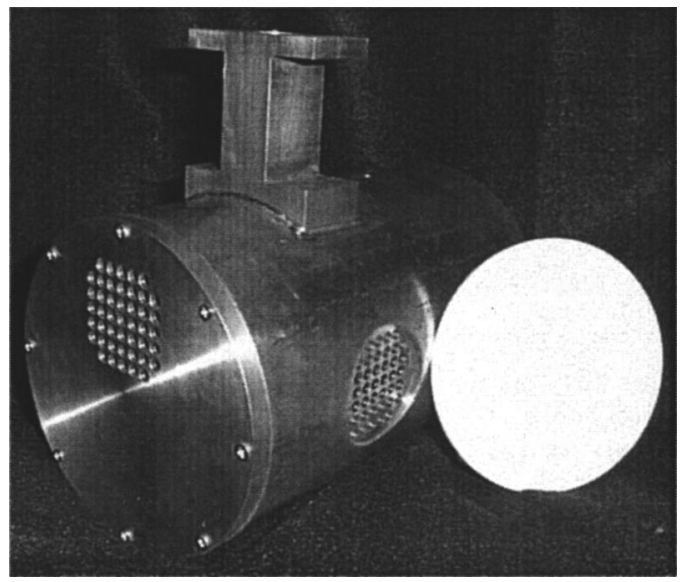

FIG. 2. Resonant cavity and dielectric: External view of cavity and the alumina disk. The external dimensions of the cavity are $\sim 15 \mathrm{~cm}$ in length and $\sim 10 \mathrm{~cm}$ in diameter. The alumina disk is $\sim 8.5 \mathrm{~cm}$ in diameter. The arrays of holes in the cavity walls are used for visual and probe access.

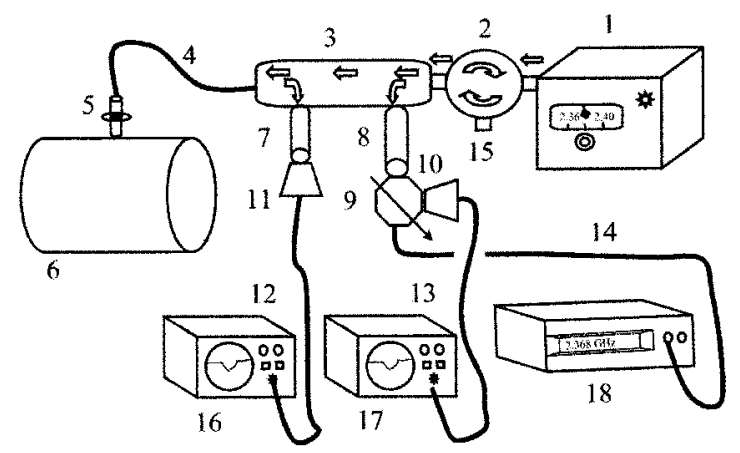

FIG. 3. Microwave circuit: (1) $2 \mathrm{~kW}$ pulsed microwave source, (2) circulator, (3) dual directional coupler, (4) type- $N$ cable, (5) microwave coupling antenna, (6) microwave resonant cavity, (7) and (8) attenuators, (9) type- $N$ switch, (10) and (11) microwave diodes, (12) and (13) BNC cables, (14) type- $N$ cable, (15) port to dummy load, (16) and (17) oscilloscopes, and (18) frequency counter.

a coupling of $\sim 20 \mathrm{~dB}$ off of the input and output port. On the reflected power arm, an attenuator of $\sim 20 \mathrm{~dB}$ is added in series with a microwave diode for reflected power measurements. The forward power arm has attenuators of $\sim 36 \mathrm{~dB}$ in series with a type- $N$ switch. The type- $N$ switch has a microwave diode and a frequency counter (Hewlett Packard, Model: 5361B) connected on two of its ports. The diode measures forward power and the frequency counter is used to tune the power supply to the microwave cavity's resonant frequency. There are three attenuators, $19 \mathrm{~dB}$ total, in series before the frequency counter for protection. The resonant frequency is measured prior to turning on the power supply from network analyzer measurements. The dual directional coupler is connected to the microwave antenna of the resonant cavity with a short type- $N$ cable. The microwave antenna is a rounded rod that couples into the electric field of the cavity. ${ }^{31}$

The diagnostics for this experiment are challenging because unlike plasma discharges in gases, there are only electrons present in this high-vacuum pulsed discharge. Ions generated as the result of outgassing do not appear in appreciable numbers during the microwave pulse. This means that conventional plasma diagnostics like optical emission spectroscopy could not be used. Probes are the dominant diagnostic for this experiment.

Holes are drilled on the cylindrical surface and ends of the cavity for probe access. A wire probe for electron detection enters the cylindrical wall of the cavity and is oriented perpendicularly to the rf electric field and parallel to the dielectric at a distance of $0.1-4 \mathrm{~cm}$ from the dielectric surface. This insures that the electric field of the $\mathrm{TE}_{111}$ mode is not strongly perturbed by the probe. The probe is connected to a dc circuit exterior to the vacuum chamber. A potentiometer, fourteen $9 \mathrm{~V}$ batteries in series, and a double throw switch allow the bias of the probe to be varied from -128 to +128 $\mathrm{V}$. The voltage across a $1.847 \mathrm{k} \Omega$ resistor is measured with an oscilloscope; this allows probe current measurements. The presence of energetic electrons made it necessary to apply several coats of carbon (Aerodag G@) to the probe in order to prevent secondary emission from the probe, which causes underestimation of the electron current. ${ }^{32}$ This electron probe can be placed at varying distances from the di- 


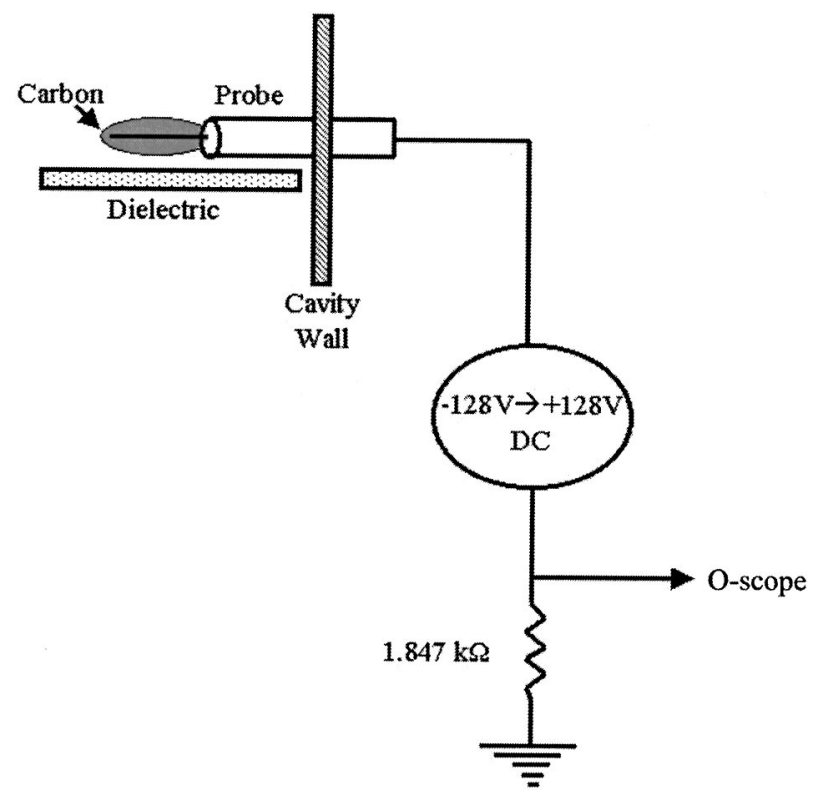

FIG. 4. Electron probe diagnostic: The electron probe provides temporal current measurements with respect to microwave forward power. Carbon was added to the probe to prevent secondary electron emission.

electric to give current measurements as a function of position, along with temporal measurements. The electron probe is illustrated in Fig. 4. The same circuit is used with a planar probe attached to the dielectric disk with conductive adhesive to provide current measurements at the dielectric surface.

Data acquisition is controlled by LABVIEW@. LABVIEW@ records the signals from all the probes and diodes from the oscilloscopes (Tektronix, Model: TDS 3052 and TDS 340) and stores the data in tab delimited format from which the signals can be plotted in an Excel@ spreadsheet.

Another diagnostic used in this experiment is electron excitation of phosphor $\left(\mathrm{Zn}_{2}: \mathrm{SiO}_{4}\right)$. Phosphor is mixed with methyl alcohol and painted in dots and lines on the surface of the alumina. This allows visual detection of multipactoring electrons on the surface of the dielectric. In order to record pictures of the flashing phosphor, the cavity was modified to allow visual access to a $35 \mathrm{~mm}$ camera. The brass end plate, opposite the alumina, was removed and replaced with a Lucite window. The Lucite window was covered with a thin wire screen of parallel wires with an orientation and separation that prevents transmission of microwaves through the screen, but at the same time allows wide-angle visual access to the inside of the cavity. When the cavity is lowered into the vacuum chamber, the Lucite window is aligned with one of the vacuum chamber windows. This allows an unobstructed path for light to reach the camera. The phosphor is a valuable diagnostic for the detection of weaker or stronger breakdown areas.

\section{RESULTS AND DISCUSSION}

Figure 5 shows temporal current measurements from the electron probe with respect to the microwave power. The power measurement is taken from the diode on the forward power arm of the dual directional coupler. The current mea-

\section{a) Microwave Forward Power Diode Signal}

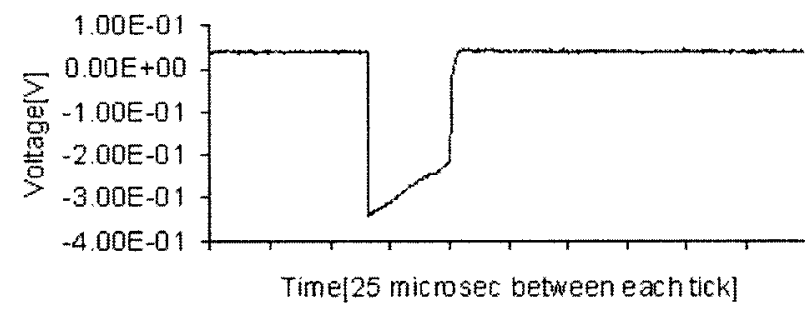

b) Electron Probe Signal

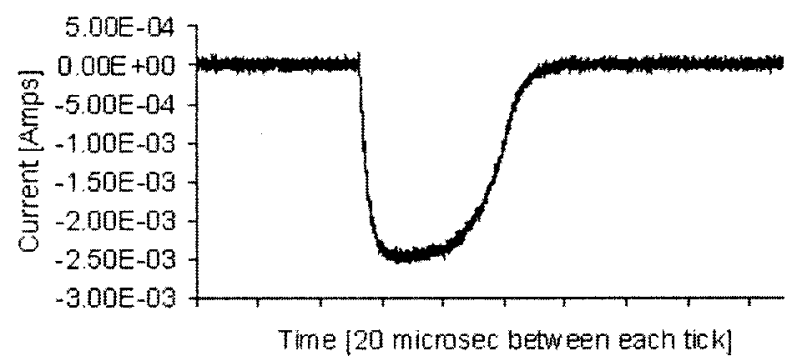

FIG. 5. Diode signals: These plots show a typical (a) forward power signal from the microwave diode and the corresponding (b) electron current. The probe was biased at $116 \mathrm{~V}$. Notice the time scale is different for the two plots.

surement is taken from the electron probe. The probe is placed $\sim 0.1 \mathrm{~cm}$ from the surface of the dielectric and is biased at $116 \mathrm{~V}$. The probe is titanium with a coating of carbon to prevent secondary emission from energetic electrons. These temporal measurements show evidence of electrons only when $\mathrm{TE}_{111}$ resonance is satisfied, and only when microwave power is on and above a threshold value. The microwave electric field at cavity resonance is measured by the bead pull perturbation method ${ }^{33-35}$ at low power and then scaled up to high power using Slater's cavity perturbation formula $^{33}$ and the low-power measured $Q$ and frequency shift $\Delta \omega$,

$$
\omega^{2}=\omega_{0}^{2}\left[1+k \frac{\int_{\Delta \tau}\left(\mu H^{2}-\epsilon E^{2}\right) \partial \tau}{\int_{V}\left(\mu H^{2}+\epsilon E^{2}\right) \partial V}\right] .
$$

$V$ is the volume of the cavity, $\Delta \tau$ is the volume of the perturbation, and $k$ is equal to unity for shapes which the fields in the cavity approach the natural fields as the volume of the object approaches zero. ${ }^{34}$ With some manipulation, a more usable relation is found

$$
E=\left[\frac{P Q \Delta \omega\left(\epsilon_{r}+2\right)}{\omega_{0}^{2} \pi r^{3} \epsilon_{0}\left(\epsilon_{r}-1\right)}\right]^{1 / 2} .
$$

where $E \equiv$ local electric field at the bead location, $P \equiv$ input power, $Q \equiv$ quality factor of cavity, $\omega_{0} \equiv$ resonant frequency, $\omega \equiv$ perturbed resonant frequency, $r \equiv$ radius of bead, $\Delta \omega$ $=\omega_{0}-\omega$, and $\epsilon_{r}=\epsilon / \epsilon_{0}$. All of these parameters are measured except $\epsilon_{r}$ and $r$, which are known from the bead manufacturer. $E_{\mathrm{rf}}$ is found to be $115 \mathrm{kV} / \mathrm{m}$ at the threshold of multipactor. An estimate of the expected threshold field can be found from the work of Kishek and Lau. ${ }^{13}$ In Fig. 4 of 
TABLE I. Comparison of theoretical $\delta_{\max }$ and tabulated $\delta_{\max }$ for alumina.

\begin{tabular}{lcc}
\hline \hline $\begin{array}{l}\text { Experimental } \\
\text { threshold } E_{\mathrm{rf}} \\
{[\mathrm{kV} / \mathrm{m}]}\end{array}$ & $\begin{array}{c}\text { Theoretical } \delta_{\max } \text { for threshold } \\
E_{\mathrm{rf}} \text { and } f=2.366 \mathrm{GHz}\end{array}$ & $\begin{array}{c}\text { Tabulated range } \\
\text { of } \delta_{\text {max }} \text { for alumina }\end{array}$ \\
\hline 115 & 6 & $1.5-9$ \\
\hline
\end{tabular}

${ }^{\mathrm{a}}$ Ref. 13 .

Kishek and Lau, ${ }^{13}$ a susceptibility curve is plotted for maximum secondary emission coefficients ranging from 1.5 to 9 . This curve gives the range of $E_{\mathrm{rf}}$ for which multipactor can occur for the possible range of secondary emission coefficient, which is used as a parameter for the curves. The $E_{\mathrm{rf}}$ is normalized with respect to frequency and the energy at the maximum secondary emission coefficient $\delta_{\max }$, thus taking into account frequency and material parameters. With our threshold $E_{\mathrm{rf}}$ of $115 \mathrm{kV} / \mathrm{m}$ and frequency of $2.366 \mathrm{GHz}$, we find from the susceptibility curve that this threshold $E_{\mathrm{rf}}$ is consistent with a $\delta_{\max }$ of approximately 6 , which is within the range usually given for alumina. ${ }^{13}$ These results are summarized in Table I.

Figure 6 is an example of the use of phosphor as an electron diagnostic. The illumination of the phosphor is an indication of the spatial distribution of electrons on the sur-

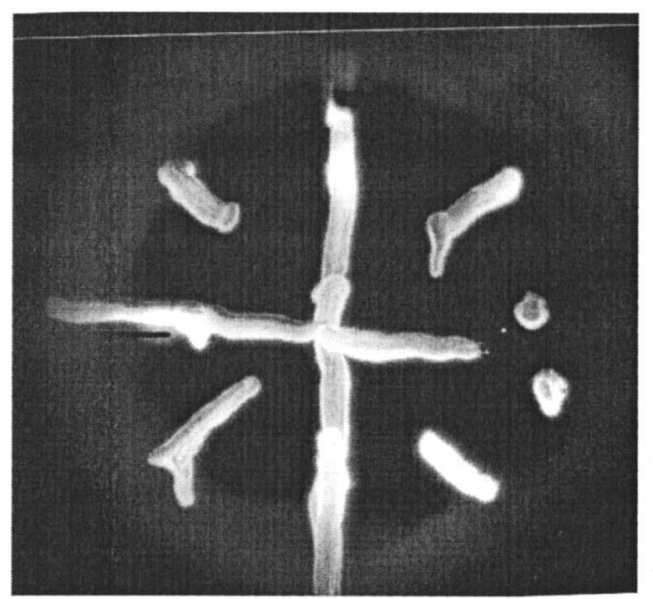

(a)

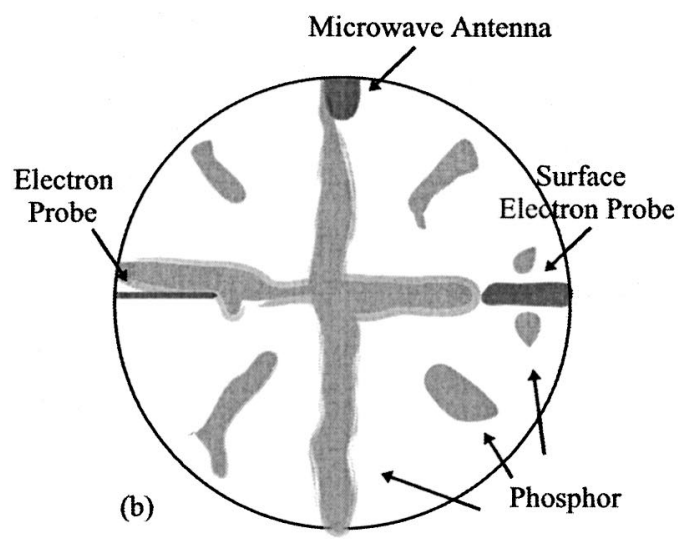

FIG. 6. Phosphor photos: (a) Photograph of phosphor diagnostic. (b) Schematic of the phosphor picture. The multipactoring electrons cause the phosphor to emit light. The two electron probes and the microwave antenna are also labeled and can be seen in the photograph. face of the dielectric. The phosphor is illuminated when the $\mathrm{TE}_{111}$ resonance is satisfied, and only when microwave power is on and above the threshold value of $E_{\mathrm{rf}}$.

The microwave resonant cavity in the $\mathrm{TE}_{111}$ mode and positioning of the dielectric duplicated the conditions that the theory predicted are necessary for multipactor to take place. Probes have provided temporal verification of electron current when the threshold field of $115 \mathrm{kV} / \mathrm{m}$ is exceeded, and phosphor gives an indication of the presence of electrons on the dielectric surface. The $E_{\mathrm{rf}}$ can be measured accurately by bead perturbation to provide comparisons with susceptibility diagrams calculated theoretically. Multipactor parameters for different materials, other than alumina, will also be explored. Using these combined measurements, the apparatus will be used to test theoretical calculations and to explore ways to prevent, or extinguish, multipactor by techniques such as tilting or coating the dielectric.

\section{ACKNOWLEDGMENT}

This work was supported by the Department of Energy, DE-FG02-98ER54475.

${ }^{1}$ D. H. Priest and R. C. Talcott, IRE Trans. Electron Devices 8, 302 (1961).

${ }^{2}$ A. D. Woode and J. Petit, ESA J. Eur. Space Agency 14, 467 (1990).

${ }^{3}$ F. Hohn, W. Jacob, R. Beckmann, and R. Wilhelm, Phys. Plasmas 4, 940 (1997).

${ }^{4}$ R. A. Kishek, Y. Y. Lau, L. K. Ang, A. Valfells, and R. M. Gilgenbach, Phys. Plasmas 5, 2120 (1998).

${ }^{5}$ R. A. Kishek, Ph.D. dissertation, University of Michigan, Ann Arbor, MI, 1997.

${ }^{6}$ R. A. Kishek and Y. Y. Lau, Phys. Rev. Lett. 75, 1218 (1995).

${ }^{7}$ A. Valfells, Ph.D. dissertation, University of Michigan, Ann Arbor, MI, 2000.

${ }^{8}$ S. Michizino et al., Vacuum 47, 625 (1996).

${ }^{9}$ A. Miura and H. Matsumoto, Nucl. Instrum. Methods Phys. Res. A 334, 341 (1993).

${ }^{10}$ M. Thumm, Int. J. Infrared Millim. Waves 19, 3 (1998).

${ }^{11}$ R. A. Rimmer, Ph.D. dissertation, University of Lancaster, UK, 1988.

${ }^{12}$ R. A. Kishek and Y. Y. Lau, Phys. Plasmas 3, 1481 (1996).

${ }^{13}$ R. A. Kishek and Y. Y. Lau, Phys. Rev. Lett. 80, 193 (1998).

${ }^{14}$ L. K. Ang, Y. Y. Lau, R. Kishek, and R. M. Gilgenbach, IEEE Trans. Plasma Sci. 26, 290 (1998).

${ }^{15}$ J. R. M. Vaughan, IEEE Trans. Electron Devices 8, 302 (1961).

${ }^{16}$ J. R. M. Vaughan, IEEE Trans. Electron Devices 15, 883 (1968).

${ }^{17}$ J. R. M. Vaughan, IEEE Trans. Electron Devices 35, 1172 (1988).

${ }^{18}$ J. R. M. Vaughan, IEEE Trans. Electron Devices 36, 1963 (1989).

${ }^{19}$ A. J. Hatch and H. B. Williams, J. Appl. Phys. 25, 417 (1954).

${ }^{20}$ A. J. Hatch and H. B. Williams, J. Appl. Phys. 32, 1086 (1961).

${ }^{21}$ R. Woo, J. Appl. Phys. 39, 1528 (1968).

${ }^{22}$ E. F. Vance, J. Appl. Phys. 34, 3237 (1963).

${ }^{23}$ A. Valfells, J. P. Verboncoeur, and Y. Y. Lau, IEEE Trans. Plasma Sci. 28, (2001)

${ }^{24}$ R. A. Kishek, Y. Y. Lau, and D. Chernin, Phys. Plasmas 4, 863 (1997).

${ }^{25}$ A. Valfells, R. A. Kishek, and Y. Y. Lau, Phys. Plasmas 5, 300 (1998).

${ }^{26}$ A. Valfells, L. K. Ang, Y. Y. Lau, and R. M. Gilgenbach, Phys. Plasmas 7, 750 (2000).

${ }^{27}$ A. Neuber, D. Hemmert, H. Krompholz, L. Hatfield, and M. Kristiansen, J. Appl. Phys. 86, 1724 (1999).

${ }^{28}$ A. Neuber, J. Dickens, D. Hemmert, H. Krompholz, L. L. Hatfield, and M. Kristiansen, IEEE Trans. Plasma Sci. 26, 296 (1998).

${ }^{29}$ A. Neuber, J. Dickens, D. Hemmert, H. Krompholz, L. L. Hatfield, M. Kristiansen, G. Cooperstein, and I. Vitkovitsky, in Proceedings of the IEEE Int. Pulsed Power Conf. 1, 135 (1997).

${ }^{30}$ Y. Saito, S. Michizono, S. Anami, and S. Kobayashi, IEEE Trans. Electr. Insul. 28, 566 (1993).

${ }^{31}$ L. Mahoney and J. Asmussen, Rev. Sci. Instrum. 61, 285 (1990). 
${ }^{32}$ E. Y. Wang, N. Hershkowitz, and D. Diebold, J. Appl. Phys. 61, 4786 (1987).

${ }^{33}$ J. C. Slater, Microwave Electronics (Van Nostrand, Princeton, NJ, 1950), p. 80 .
${ }^{34}$ E. L. Ginzton, Microwave Measurements (McGraw-Hill, York, PA, 1957), p. 448.

${ }^{35}$ R. F. Harrington, Time-Harmonic Electromagnetic Fields (McGraw-Hill, 1961), p. 317. 